\title{
Utilización potencial de una ceniza volante de baja calidad como fuente de aluminosilicatos en la producción de geopolímeros'
}

\section{Potential Utilization of a Low Quality-Fly Ash as an Aluminosilicate Source in the Production of Geopolymers ${ }^{2}$}

\author{
Johanna M. Mejía ${ }^{3}$ \\ Erich D. Rodríguez ${ }^{4}$ \\ Ruby Mejía de Gutiérrezs
}

doi:10.11144/Javeriana.IYU18-2.upcv

Cómo citar este artículo:

MEJÍA J. M.; RODRÍGUEZ, E. D. y MEJÍA DE GUTIÉRREZ, R. Utilización potencial de una ceniza volante de baja calidad como fuente de aluminosilicatos en la producción de geopolímeros. Ingeniería y Universidad. 2014, vol. 18, no. 2, pp. 309-327. http://dx.doi.org/10.11144/Javeriana.IYU18-2.upcv

\footnotetext{
'Fecha de recepción: 18 de julio de 2013. Fecha de aceptación: 31 de julio de 2014. Este artículo se deriva de un proyecto de investigación denominado Desarrollo de un nuevo cemento híbrido tipo Portland con alto porcentaje de adición, desarrollado por el grupo de investigación Materiales Compuestos de la Universidad del Valle, Cali, Colombia.

${ }^{2}$ Reception date: July $18^{\text {th }}$, 2013. Acceptance date: July 31 st, 2014. This article is derived from the research project Development of a novel hybrid cement with higher content of the addition, developed by the research group Composites Materials, Universidad del Valle, Cali, Colombia.

${ }^{3}$ Estudiante de Doctorado en Ingeniería. Miembro del Grupo Materiales Compuestos (CENM), Universidad del Valle, Cali, Colombia. Correo electrónico: johanna.mejia@correounivalle.edu.co

${ }^{4}$ Doctor en Ingeniería. Miembro del Grupo Materiales Compuestos (CENM), Universidad del Valle, Cali, Colombia. Correo electrónico: erich.rodriguez@correounivalle.edu.co

${ }^{5}$ Doctora en Ciencias Químicas. Miembro del Grupo Materiales Compuestos (CENM), Universidad del Valle, Cali, Colombia. Correo electrónico: ruby.mejia@correounivalle.edu.co
} 


\section{Resumen}

Este artículo presenta la síntesis de materiales geopoliméricos a partir de una ceniza volante de origen colombiano (FA). Se evaluaron diferentes condiciones de activación de esta ceniza mediante el ajuste de los módulos totales de $\mathrm{SiO}_{2} / \mathrm{Al}_{2} \mathrm{O}_{3}$ y Na${ }_{2} \mathrm{O} / \mathrm{SiO}_{2}$, así como el efecto de la incorporación de dos fuentes de calcio: una escoria granulada de alto horno (GBFS) y un cemento Portland tipo I (OPC). La cinética de las reacciones desarrolladas después de la activación alcalina de la FA se evaluó realizando una calorimetría isotérmica, según la cual los sistemas de mayor alcalinidad exhiben una mayor liberación de calor y un mayor grado de disolución de la fuente primaria. La incorporación de la fuente de calcio modifica la cinética de reacción y el tipo y características de los productos formados tras la activación. El incremento en la relación molar $\mathrm{SiO}_{2} /$ $\mathrm{Al}_{2} \mathrm{O}_{3}$ del sistema condujo a obtener materiales con una resistencia a la compresión superior hasta en $\sim 77 \%$. En términos generales, la incorporación de una fuente de calcio al sistema contribuye a la adquisición de un mejor desempeño mecánico en todas las edades de curado en estudio. Los resultados evidencian el potencial aprovechamiento de esta FA para producir cementos no convencionales con resistencias a 28 días de curado superiores a los $50 \mathrm{MPa}$.

\section{Palabras clave}

geopolímeros; ceniza volante; escoria granulada de alto horno; calorimetría; cementos no convencionales

\section{Abstract}

This article presents the synthesis of a geopolymeric material based on a Colombian fly ash (FA). Geopolymers with different total molar $\mathrm{SiO}_{2} / \mathrm{Al}_{2} \mathrm{O}_{3}$ and $\mathrm{Na}_{2} \mathrm{O} / \mathrm{SiO}_{2}$ ratios and the inclusion of two source of calcium (a national granulated blast furnace slag and ordinary Portland cement) were evaluated. The reaction kinetic of FA alkali activation was monitored by isothermal calorimetry, which shows the systems with highest alkalinity exhibit higher release of heat and higher dissolution degree of FA. Replacing a percentage of FA by a calcium source (OPC or GBFS) modifies the kinetic and reaction products. A higher $\mathrm{SiO}_{2} /$ $\mathrm{Al}_{2} \mathrm{O}_{3}$ molar ratio leads to the production of materials with higher compressive strength up to $\sim 77 \%$. Overall, the addition of a calcium source contributes significantly to the acquisition of a higher mechanical performance. The results show the potential use of this FA with unprofitable physical and chemical characteristics as a raw material to produce non-conventional cementitious with compressive strength up to $50 \mathrm{MPa}$ at 28 days of curing time.

\section{Keywords}

geopolymers; fly ash; granulated blast furnace slag; isothermal calorimetry; unconventional cements 


\section{Introducción}

La Asociación Mundial del Carbón (2014) reporta que el principal combustible utilizado a escala mundial para generar electricidad es el carbón, con un consumo del $41 \%$ en el sector energético, en 2012. Sin embargo, el uso de este recurso natural como fuente energética genera diferentes subproductos o desechos, como cenizas de fondo, escoria, cenizas volantes (FA), entre otros productos. Las (FA) corresponden a la parte inorgánica del carbón remanente del proceso de combustión entre los 1200 y los $1700{ }^{\circ} \mathrm{C}$ y están compuestas principalmente por minerales no combustibles que quedan en la cámara de combustión en forma de finas cenosferas. Estas partículas son conducidas por ductos de gases hacia filtros o ciclones encargados de capturar el material y evitar que sean emitidas a la atmósfera (Ahmaruzzaman, 2010). En Colombia, las termoeléctricas más importantes (Termozipa, Termopaipa y Termosochagota) generan alrededor de 600 kt/año de (FA) de calidad variable y en su gran mayoría este subproducto no se está aprovechando debidamente y se constituye en un problema ambiental (Gil y Plazas, 2007).

Las FA se caracterizan por ser ricas en $\mathrm{SiO}_{2}, \mathrm{Al}_{2} \mathrm{O}_{3}$ y, en algunos casos, presenta un elevado contenido de $\mathrm{CaO}$, así como trazas de $\mathrm{Fe}_{2} \mathrm{O}_{3}$ y otros óxidos. En la industria de la construcción, las FA se destinadan como materia prima en la producción del cemento Portland, adición activa en la producción de cementos adicionados (blended cements), en la producción in situ de concretos o como material de relleno en la estabilización de suelos, entre otros (Gil y Plazas, 2007). La norma ASTM C618-12a clasifica las FA en dos tipos: clase C (cenizas ricas en $\mathrm{CaO}$ ) y clase $\mathrm{F}$ (cenizas bajas en $\mathrm{CaO}$ ), y establece los criterios para su utilización como material puzolánico en mezclas con cemento portland. Entre estos se exige un porcentaje de pérdidas por ignición inferior al $6 \%$. Se afirma que cenizas con valores superiores pueden conducir a una mayor demanda de agua en las mezclas que la contengan y, con ello, un decremento en la trabajabilidad en estado fresco, una gran variabilidad en las resistencias mecánicas, así como un efecto negativo en las propiedades de durabilidad (Xu et al., 2012). 
En los últimos años, y debido a su composición de tipo aluminosilicato, las FA han venido utilizándose como materia prima en la fabricación de cementantes no convencionales simples y binarios, libres de cemento Portland, denominados geopolímeros (Temuujin, Rickard y van Riessen, 2013; van Deventer, Provis y Duxson, 2012), y aunque se han reportado resistencias mecánicas hasta de $100 \mathrm{MPa}$, la mayoría de los estudios se basan en cenizas que cumplen ampliamente las características de calidad señaladas en la norma ASTM C618 (van Riessen y Chen-Tan, 2013; Nematollahi y Sanjayan, 2014; Ryu et al., 2013; Kumar, Kumar y Mehrotra, 2007; Fernández-Jiménez et al., 2011; Izquierdo et al., 2009; Yip et al., 2008; Puertas y Fernández-Jiménez, 2003).

Un estudio realizado para evaluar el efecto de las características físicas y químicas de cenizas procedentes de diferentes fuentes en las propiedades de resistencia mecánica concluye que para una activación alcalina óptima, las FA deben poseer un contenido alto de fase vítrea, un $80 \%$ de partículas de tamaño inferior a $45 \mu \mathrm{m}$ y un porcentaje inferior al $5 \%$ de pérdidas por ignición (Fernández-Jiménez y Palomo, 2003). Como opciones para reducir el nivel de carbón presente en la ceniza, previo a la síntesis de un geopolímero, se han considerado el tratamiento térmico y los métodos de flotación. De estos, el primero reduce la fase vítrea presente y afecta negativamente las propiedades finales del material y el segundo aunque actúa positivamente implica costos asociados a un proceso adicional (Temuujin y van Riessen, 2009; Lee et al., 2010).

El presente estudio plantea una alternativa para valorizar y aprovechar una FA de origen nacional con un alto contenido de pérdidas por ignición $(14,6 \%)$ y un bajo porcentaje de fase vítrea. Este material no presenta un valor comercial atractivo para su aplicación en la industria cementera o de concretos y actualmente es catalogado como un desecho industrial con sus respectivos inconvenientes para su correcta disposición. Por ello, el objetivo principal es evaluar su aplicación potencial en la producción de materiales geopoliméricos y, de esta manera, otorgarle un valor agregado. Con este fin se producen geopolímeros basados en un $100 \%$ FA y geopolímeros binarios que incorporan una fuente de calcio - escoria granulada de alto horno (GBFS) y cemento Portland tipo I (OPC)—. Los materiales obtenidos se caracterizaron mediante la determinación de la resistencia a compresión y la aplicación de diferentes técnicas instrumentales, como calorimetría isotérmica y microscopía electrónica de barrido. 


\section{Metodología experimental}

\subsection{Materiales}

Como precursor de aluminosilicatos se empleó una FA proveniente de la central termoeléctrica de Termopaipa. La FA con un tamaño medio de partícula de $\sim 64 \mu \mathrm{m}$ y una distribución de tamaño de partículas donde el $90 \%$ es inferior a $141,3 \mu \mathrm{m}$, fue sometida a un tratamiento de molienda en un molino de bolas durante $90 \mathrm{~min}$. El material obtenido presentó un tamaño medio de partícula de $19,5 \mu \mathrm{m}$ y un peso específico de $2216 \mathrm{~kg} / \mathrm{m}^{3}$. Como fuentes adicionales de calcio se empleó una GBFS proveniente de Acerías Paz del Río (Colombia) y un OPC con un peso específico de $3200 \mathrm{~kg} / \mathrm{m}^{3}$. La composición química de los materiales se presenta en la tabla 1 . Se aprecia un contenido elevado de pérdida al fuego $(14,8 \%)$ para FA como consecuencia del material orgánico presente (carbón no quemado); así mismo, el OPC reporta un 9,6\% de pérdidas por ignición. Esto se atribuye a la adición caliza que incluye este material. Acorde a los datos de composición química, la relación molar $\mathrm{SiO}_{2} / \mathrm{Al}_{2} \mathrm{O}_{3}$ de $\mathrm{FA}$ es 4,25.

Tabla l. Composición química de las materias primas

\begin{tabular}{|c|c|c|c|}
\hline \multirow{2}{*}{ Compuesto } & FA & GBFS & $O P C$ \\
\hline & \multicolumn{3}{|c|}{ ( $\%$ en peso) } \\
\hline $\mathrm{SiO}_{2}$ & 53,7 & 32,0 & 20,2 \\
\hline $\mathrm{Al}_{2} \mathrm{O}_{3}$ & 21,5 & 14,5 & 7,0 \\
\hline $\mathrm{Fe}_{2} \mathrm{O}_{3}$ & 4,5 & 1,1 & 4,8 \\
\hline $\mathrm{CaO}$ & 0,8 & 46,9 & 58,4 \\
\hline $\mathrm{TiO}_{2}$ & 1,0 & 0,5 & - \\
\hline $\mathrm{K}_{2} \mathrm{O}$ & 1,4 & 0,4 & - \\
\hline$S$ & 0,6 & 0,8 & - \\
\hline $\mathrm{MgO}$ & 0,6 & 1,0 & - \\
\hline $\mathrm{P}_{2} \mathrm{O}_{5}$ & 0,5 & 0,2 & - \\
\hline $\mathrm{Na}_{2} \mathrm{O}$ & 0,3 & 0,2 & - \\
\hline $\mathrm{MnO}$ & - & 0,4 & - \\
\hline $\mathrm{Sr}$ & 0,2 & 0,1 & - \\
\hline Pérdida al fuego $\left(950{ }^{\circ} \mathrm{C}\right)$ & 14,8 & 1,8 & 9,6 \\
\hline
\end{tabular}

Fuente: presentación propia de los autores 
El difractograma de FA (figura 1), realizado en un difractómetro X X Pert MRD de PanAnalytical usando radiación de $\mathrm{Cu} K \alpha 1$ con un tamaño de paso de $0,020^{\circ}$, revela la presencia de diferentes fases cristalinas como: mullita $\left(2 \mathrm{Al}_{2} \mathrm{O}_{3}\right.$ $\mathrm{SiO}_{2}$; American Mineralogist Crystal Structure Database, AMSCD 0001059), hematita $\left(\mathrm{Fe}_{2} \mathrm{O}_{3}\right.$; AMSCD 000143) y cuarzo $\left(\mathrm{SiO}_{2} ; 0000789\right)$. El correspondiente para GBFS reveló la presencia de calcita $\left(\mathrm{CaCO}_{3}\right.$; $\mathrm{AMSCD}$ 0000098), gelenita $\left(\mathrm{Ca}_{2} \mathrm{Al}_{[} \mathrm{AlSiO}_{7}\right] ;$ AMSCD 00005090) y cuarzo ( $\mathrm{SiO}_{2} ; \mathrm{AMSCD}$ 0000789). Como activadores alcalinos se empleó la mezcla de un hidróxido de sodio grado analítico ( $\sim 99 \%$ ) y un silicato de sodio comercial constituido con un 26,50\% de $\mathrm{SiO}_{2}$; un $7,93 \%$ de $\mathrm{Na}_{2} \mathrm{O}$, y un $65,57 \%$ de $\mathrm{H}_{2} \mathrm{O}$ para la obtención de soluciones con módulos $\left(\mathrm{Ms}=\mathrm{SiO}_{2} / \mathrm{Na}_{2} \mathrm{O}\right)$ entre 0,6 y 1,5 .

\subsection{Preparación de las muestras}

Se produjeron sistemas simples basados exclusivamente en FA y sistemas binarios constituidos por la mezcla de FA y una fuente adicional de calcio (GBFS o OPC). Las fuentes de Ca se incorporaron con base en un $30 \%$ en sustitución de FA. La activación de FA fue realizada mediante la dosificación de un activador alcalino cuyas proporciones permitieron obtener una relación molar total de $\mathrm{SiO}_{2} / \mathrm{Al}_{2} \mathrm{O}_{3}$ de 5 y 6 , así como $\mathrm{Na}_{2} \mathrm{O} / \mathrm{SiO}_{2}$ de 0,21 y 0,25 . El contenido de agua fue ajustado para obtener una relación agua/conglomerante de 0,35 .

La FA, la fuente de Ca y el activador alcalino fueron mezclados mecánicamente y posteriormente vaciados en moldes cúbicos de $20 \times 20 \mathrm{~mm}$. Las muestras FA/ GBFS y FA/OPC fueron almacenadas en una cámara húmeda a temperatura ambiente hasta el momento de los ensayos. Los especímenes constituidos únicamente con FA (es decir, sin la inclusión de una fuente de $\mathrm{Ca}$ ) requirieron la utilización de un curado térmico a $70{ }^{\circ} \mathrm{C}$ con una $\mathrm{HR}$ del $90 \%$ durante $24 \mathrm{~h}$. Las condiciones anteriores se apoyan en estudios reportados por distintos autores (Kovalchuk, Fernández-Jiménez y Palomo, 2008; Fernández-Jiménez, Palomo y Criado, 2005). Al finalizar el curado térmico las muestras fueron almacenadas en una cámara bajo condiciones de humedad relativa del $90 \%$. En la tabla 2 se muestran las características de las mezclas producidas. 
Figura 1. Difractogramas de la ceniza (FA) y la escoria granulada de alto horno (GBFS)

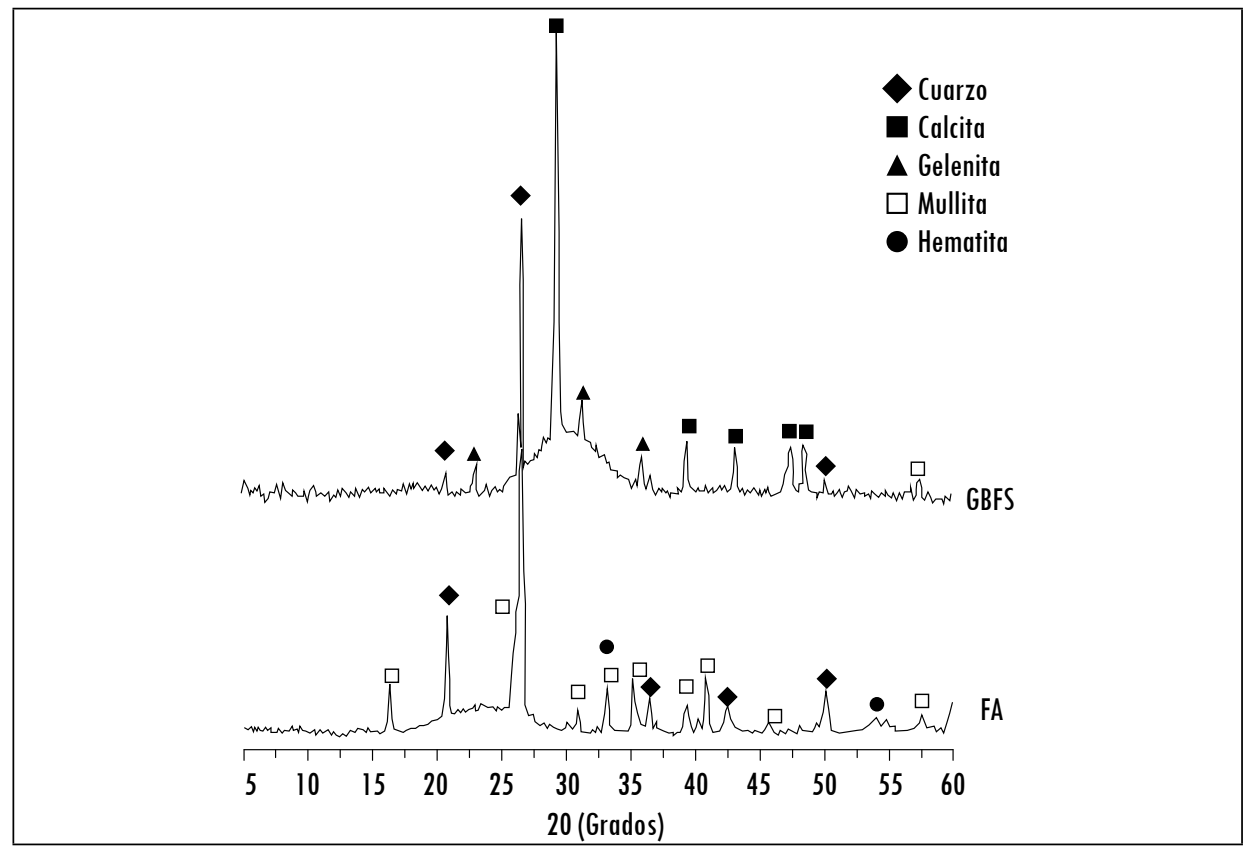

Fuente: presentación propia de los autores

Tabla 2. Mezclas geopoliméricas producidas

\begin{tabular}{|l|c|c|c|c|c|}
\hline \multirow{2}{*}{ Referencia de la mezcla } & \multicolumn{2}{|c|}{$\begin{array}{c}\text { Contenido de la fuente } \\
\text { de Ca (\%)* }\end{array}$} & \multirow{2}{*}{ SiO $_{2} / \mathrm{Al}_{2} \mathbf{O}_{3}$} & $\mathrm{Na}_{2} \mathbf{O} / \mathrm{SiO}_{2}$ & \multirow{2}{*}{$\mathbf{W} / \mathbf{b}$} \\
\cline { 2 - 3 } & GBFS & $\mathbf{O P C}$ & & & \\
\hline FA-5-21 & -- & -- & 5,0 & 0,21 & 0,35 \\
\hline FA-5-25 & -- & -- & 5,0 & 0,25 & 0,35 \\
\hline FA-6-21 & -- & -- & 6,0 & 0,21 & 0,35 \\
\hline FA-6-25 & -- & -- & 6,0 & 0,25 & 0,35 \\
\hline FA-30GBFS-5-21 & 30 & -- & 5,0 & 0,21 & 0,35 \\
\hline FA-30GBFS-5-25 & 30 & -- & 5,0 & 0,25 & 0,35 \\
\hline FA-30GBFS-6-21 & 30 & -- & 6,0 & 0,21 & 0,35 \\
\hline FA-30GBFS-6-25 & 30 & -- & 6,0 & 0,25 & 0,35 \\
\hline FA-30OPC-5-21 & -- & 30 & 5,0 & 0,21 & 0,35 \\
\hline FA-30OPC-5-25 & -- & 30 & 5,0 & 0,25 & 0,35 \\
\hline FA-30OPC-6-21 & -- & 30 & 6,0 & 0,21 & 0,35 \\
\hline FA-30OPC-6-25 & -- & 30 & 6,0 & 0,25 & 0,35 \\
\hline
\end{tabular}

*Porcentaje en peso respecto a la totalidad del precursor.

w: corresponde al agua contenida en el activador alcalino y el agua libre; $b$ : hace referencia a los precursores y la fracción anhidra del activador

Fuente: presentación propia de los autores 


\subsection{Ensayos realizados}

- Calorimetría isotérmica: el proceso de reacción de cada uno de los sistemas se evaluó usando la técnica de calorimetría isotérmica mediante un calorímetro de Calmetrix I-cal 8000 a una temperatura de $25^{\circ} \mathrm{C}$ durante $40 \mathrm{~h}$. Las mezclas se prepararon manualmente fuera del calorímetro y el registro del cambio de calor se inició 4 min después de haber iniciado el mezclado de los componentes.

- Resistencia a compresión: los especímenes fueron ensayados después de 7 y 28 días de curado en una prensa Universal INSTRON 3369 a una velocidad de desplazamiento de $1 \mathrm{~mm} / \mathrm{min}$.

- Caracterización microestructural: complementariamente, se llevó a cabo una observación mediante microscopía electrónica de barrido de algunas de las muestras previamente evaluadas. Se empleó un microscopio electrónico Phenom 2005 de FEI con un voltaje de aceleración de 5 kV.

\section{Resultados y discusión}

\subsection{Calorimetría isotérmica de conducción}

La figura 2 muestra las curvas calorimétricas para los diferentes sistemas geopoliméricos estudiados. La figura 2 a corresponde a la curva calorimétrica de las mezclas constituidas con un $100 \%$ de FA, donde se identifica un único pico: este, en general, se presenta en un tiempo inferior a $10 \mathrm{~min}$. La intensidad del pico varía en función de las condiciones de activación, pero alcanza su valor máximo $(23,23 \mathrm{~mW} / \mathrm{g})$ para los sistemas con una relación $\mathrm{SiO}_{2} / \mathrm{Al}_{2} \mathrm{O}_{3}$ de 5 . Estos resultados son superiores a los reportados por cementos tradicionales, los cuales pueden estar entre 3,5 y $9 \mathrm{~mW} / \mathrm{g}$ (Xu et al., 2010; Gruyaert, Robeyst y De Belie, 2010). El cambio en el módulo $\mathrm{Na}_{2} \mathrm{O} / \mathrm{SiO}_{2}$ de 0,21 a 0,25 incrementa la intensidad del pico de liberación de calor de hasta $\sim 7,1 \mathrm{~mW}$, lo que puede atribuirse a un mayor grado de disolución de la FA, como consecuencia del aumento en el contenido de álcalis en la pasta.

Una mayor alcalinidad del sistema contribuye a la precipitación de los productos formados y los picos atribuidos a la preinducción, a la inducción y a la aceleración (los cuales son característicos en sistemas de OPC) no son identificados individualmente, debido a que las reacciones de disolución y precipitación se desarrollan simultáneamente. García-Lodeiro, Fernández-Jiménez y Palomo (2013), en un estudio similar, no observan la aparición de este pico y, por tanto, reporta que, aun cuando la presencia de álcalis induce el inicio de la disolución de la ceniza, este proceso a temperatura ambiente es extremadamente lento y, 
en consecuencia, la precipitación del gel de aluminosilicato $(\mathrm{N}-\mathrm{A}-\mathrm{S}-\mathrm{H})$ se reduce notablemente; por ello es necesario acelerar la reacción para que este proceso sea tecnológicamente viable. En el presente estudio se corroboró que la mezcla de FA al $100 \%$, independientemente de las relaciones de mezcla utilizadas, no fragua ni endurece a temperatura ambiente, por ello se aplicó un curado térmico $\left(70^{\circ} \mathrm{C}\right.$, HR $90 \%, 24$ h).

Figura 2. Calor liberado en mezclas geopoliméricas: a) basadas únicamente con ceniza volante y los sistemas constituidos con b) escoria y c) cemento Portland
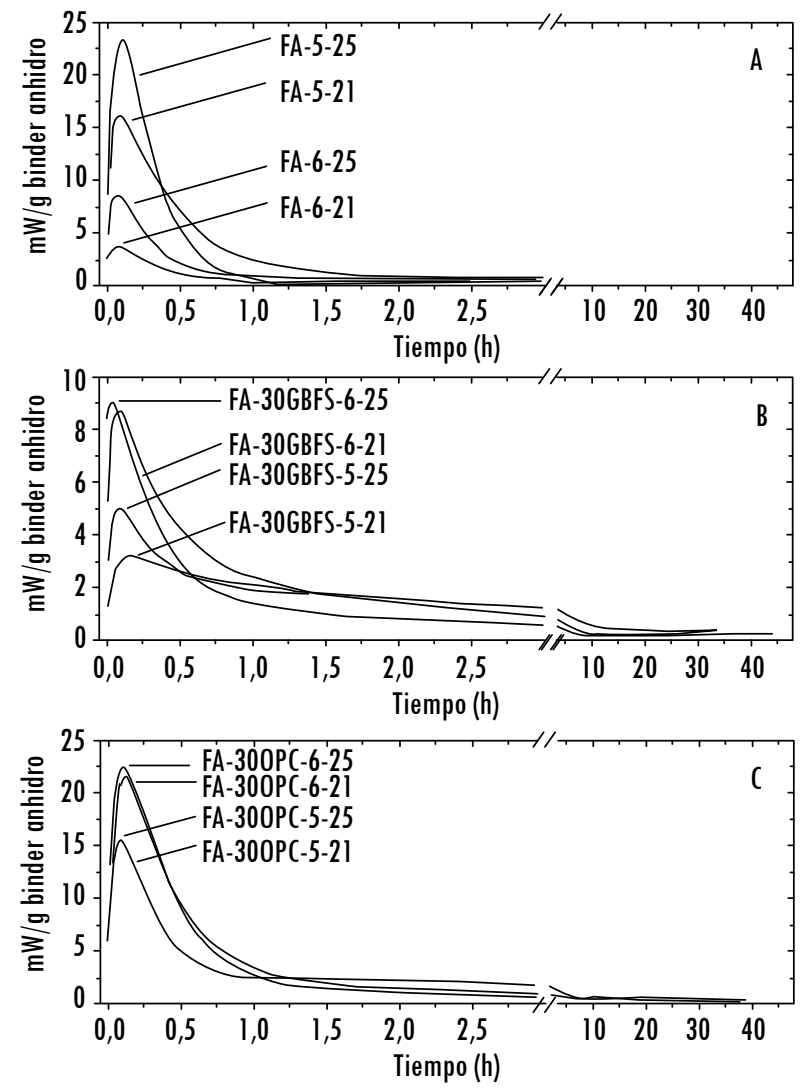

Fuente: presentación propia de los autores

La incorporación de una fuente de Ca tiene un efecto en la liberación de calor, en la cinética de reacción y en las características de los productos formados. Así, la incorporación de GBFS condujo a una reducción en la liberación de 
calor (figura 2b), mientras que el OPC presentó una leve disminución en los picos máximos en función de la relación $\mathrm{SiO}_{2} / \mathrm{Al}_{2} \mathrm{O}_{3}$ de cada sistema. Tal como se puede apreciar en las figuras $2 \mathrm{~b}$ y $2 \mathrm{c}$, los sistemas con un módulo $\mathrm{SiO}_{2} / \mathrm{Al}_{2} \mathrm{O}_{3}$ de 6, e independientemente del tipo de fuente de Ca utilizada, exhiben una mayor liberación de calor, contrario a lo identificado en las muestras constituidas únicamente con FA (figura 2a).

En términos generales, no se identifican grandes diferencias con el incremento en el módulo $\mathrm{Na}_{2} \mathrm{O} / \mathrm{SiO}_{2}$ para los sistemas con OPC y GBFS. A pesar de que los valores máximos de calor liberado para los sistemas FA/OPC son más bajos, comparados con los sistemas basados únicamente con FA; los FA/OPC exhiben los valores más altos de calor total, seguidos de los sistemas FA/GBFS, tal como se puede identificar en la tabla 3. Este comportamiento indica que los sistemas constituidos con una fuente de Ca conducen a un mayor grado de reacción como consecuencia de la mayor solubilidad por parte del OPC y la GBFS en el entorno alcalino, así como la precipitación de un mayor contenido de productos de reacción. Estos resultados son coherentes con lo reportado por Kumar, Kumar y Mehrotra (2010), quienes identificaron el aumento en la cantidad de calor total liberado conforme se incrementaba el contenido de GBFS en un sistema binario con FA activado a $27^{\circ} \mathrm{C}$. Este comportamiento está íntimamente relacionado con el no requerimiento de curado térmico para estas mezclas, las cuales fraguan y endurecen a temperatura ambiente.

Tabla 3. Calor total liberado en los geopolímeros durante las primeras 40 h de reacción

\begin{tabular}{|l|c|}
\hline \multicolumn{1}{|c|}{ Mezcla } & Calor total liberado $\left(Q^{\prime}{ }_{\max }\right) \mathbf{k J} / \mathbf{k g}$ \\
\hline FA-5-21 & 76,27 \\
\hline FA-5-25 & 44,82 \\
\hline FA-6-21 & 31,53 \\
\hline FA-6-25 & 50,86 \\
\hline FA-30GBFS-5-21 & 87,72 \\
\hline FA-30GBFS-5-25 & 87,96 \\
\hline FA-30GBFS-6-21 & 73,75 \\
\hline FA-30GBFS-6-25 & 75,41 \\
\hline FA-30OPC-5-21 & 105,70 \\
\hline FA-30OPC-5-25 & 103,79 \\
\hline FA-30OPC-6-21 & 92,11 \\
\hline FA-30OPC-6-25 & 100,03 \\
\hline
\end{tabular}

Fuente: presentación propia de los autores 
La aparición de un único pico de elevada intensidad coincide con lo presentado en otros sistemas de activación alcalina basados en FA o GBFS y sus correspondientes mezclas (Bernal et al., 2011a; Chithiraputhiran y Neithalath, 2013; Winnefeld et al., 2010). Chithiraputhiran y Neithalath (2013) reportaron, en mezclas geopoliméricas FA:GBFS 70:30, curvas calorimétricas similares a las aquí presentadas, y un calor total liberado del orden de $60 \mathrm{~kJ} / \mathrm{kg}$ a 40 h de reacción. García-Lodeiro, Fernández-Jiménez y Palomo (2013) reportaron, igualmente, valores similares para mezclas geopoliméricas FA:OPC 70:30. Estos resultados son inferiores hasta en un $40 \%$ a los obtenidos en el presente estudio para las mezclas conteniendo GBFS y OPC (tabla 3). En general, la mayor liberación de calor en estos sistemas se da en las primeras horas y se atenúa o estabiliza rápidamente. Este comportamiento difiere del proceso de hidratación del cemento Portland, donde la liberación del calor aumenta en el tiempo (García-Lodeiro, Fernández-Jiménez y Palomo, 2013).

La geopolimerización inicia con la disolución del precursor, seguido de una precipitación de especies activas (monómeros de Si y Al), los cuales sufren una policondensación para la formación de oligómeros que posteriormente polimerizan formando el gel N-A-S-H (Fernández-Jiménez, Palomo y Criado, 2005; Provis y van Deventer, 2009). Durante las primeras horas, la precipitación y la condensación de especies de monómeros y dímeros obtenidos durante la disolución del precursor conducen a la formación de un gel $\mathrm{N}-\mathrm{A}-\mathrm{S}-\mathrm{H}$ rico en $\mathrm{Al}$ con una estructura tipo $\mathrm{Q}^{4}(4 \mathrm{Al})$ (Puertas et al., 2011). La amplitud del pico puede deberse al tiempo requerido para que las especies iónicas en el sistema alcancen una concentración crítica para formar los productos de reacción (Provis y van Deventer, 2007).

Conforme se desarrolla la reacción, este gel se transforma en uno más estable rico en tectosilicatos con un efecto positivo en el desempeño mecánico del material como consecuencia de la presencia de los silicatos disueltos que son incorporados en el activador (Duxson et al., 2007). La sílice presente en la solución activante es muy soluble y es incorporada en el gel N-A-S-H. No obstante, el grado de polimerización de la sílice en la solución de silicato, el cual depende de la relación $\mathrm{SiO}_{2} / \mathrm{Na}_{2} \mathrm{O}$, condiciona la formación y características estructurales del gel N-A-S-H y con ello la cantidad de calor liberado durante la reacción. La mayor liberación de calor puede atribuirse a la presencia de concentraciones de alcalinidad mayores en el sistema (Ravikumar y Neithalath, 2012). El producto final es un gel N-A-S-H con una estructura tridimensional de unidades $\mathrm{Q}^{4}(\mathrm{mAl})$ (con m: 1,2 o 3), cuyas características dependen del grado de reacción, de la 
temperatura de curado y del contenido de silicatos solubles en el sistema (Duxson et al., 2005a y 2005b).

La incorporación de una fuente de Ca promueve la formación de otro tipo de productos, fundamentalmente $\mathrm{C}-(\mathrm{A})-\mathrm{S}-\mathrm{H}$ y $\mathrm{C}-\mathrm{S}-\mathrm{H}$, con una cinética y grado de reacción diferente a lo presentado por el gel N-A-S-H y que coexiste con el gel geopolimérico (García-Lodeiro, Fernández-Jiménez y Palomo, 2013). Mediante un análisis de microscopía electrónica de barriso para mezclas $70 \% \mathrm{FA}+30 \% \mathrm{OPC}$ se comprobó la coexistencia de dos geles C-(A)-S-H y N-A-S-H, este último con pequeñas cantidades de calcio (N,C)-A-S-H. Estos productos, en general, tienen un efecto positivo en las resistencias mecánicas del material (García-Lodeiro et al., 2010; Bernal et al., 2011b), donde las características estructurales dependen de igual manera del tipo de fuente de Ca utilizada (Yip et al., 2008). Este efecto es más marcado a elevadas relaciones $\mathrm{SiO}_{2} / \mathrm{Na}_{2} \mathrm{O}$ o en sistemas con baja alcalinidad (Alonso y Palomo, 2001).

\subsection{Resistencia a compresión}

La figura 3 a muestra la evolución de la resistencia a compresión de los sistemas constituidos exclusivamente con FA, en la cual se aprecia un incremento de entre el $3 \%$ y el $18 \%$, conforme avanza la edad de curado y cuyo valor máximo (28 MPa) fue alcanzado por FA 6-21 después de 28 días. En la figura $3 \mathrm{~b}$ se muestran los resultados obtenidos para los sistemas binarios FA-GBFS y FA-OPC, donde se observó un incremento de la resistencia en comparación con los sistemas basados en FA, así como un mayor aumento de la resistencia conforme avanza la edad de curado, que alcanza valores hasta de $\sim 55 \mathrm{MPa}$.

Los reducidos incrementos de resistencia con la edad de curado reportados por los sistemas basados en FA (figura 3a) pueden atribuirse al curado térmico previamente aplicado, el cual contribuye a adquirir un elevado grado de reacción a tempranas edades que no conduce a la formación de nuevos productos, densificación estructural y, con ello, incrementos considerables en la resistencia mecánica a tiempos más prolongados (Puertas, Martínez-Ramírez y Vázquez, 2000). Por su parte, en los sistemas constituidos con una fuente de Ca (OPC y GBFS), la formación de otro tipo de estructuras, tal como se mencionó en el apartado anterior, densifican y aumentan su grado de reticulación con el tiempo y se generan incrementos de resistencias de hasta un $116 \%$ a edades más avanzadas. Cabe anotar que estos sistemas son curados a temperatura ambiente. El incremento de la resistencia es coherente con otros reportes (Puertas, MartínezRamírez y Vázquez, 2000; Phoo-ngernkham et al., 2013). Existe una relación 
entre el calor total liberado y los valores de resistencia a compresión obtenidos, donde los sistemas simples (basados únicamente con FA); además de presentar las resistencias mecánicas más bajas, liberaron la menor cantidad de calor durante su activación. Por otra parte, la incorporación de una fuente de Ca (ya sea OPC o GBFS), aparte de contribuir al incremento en la cantidad total de calor liberado (tabla 3), genera mayores resistencias mecánicas en el sistema.

Figura 3. Resistencia a compresión de los sistemas geopoliméricos: a) sistema simple únicamente ceniza volante: $F A$; b) sistemas binarios ricos en calcio: FA-GBFS y FA-OPC
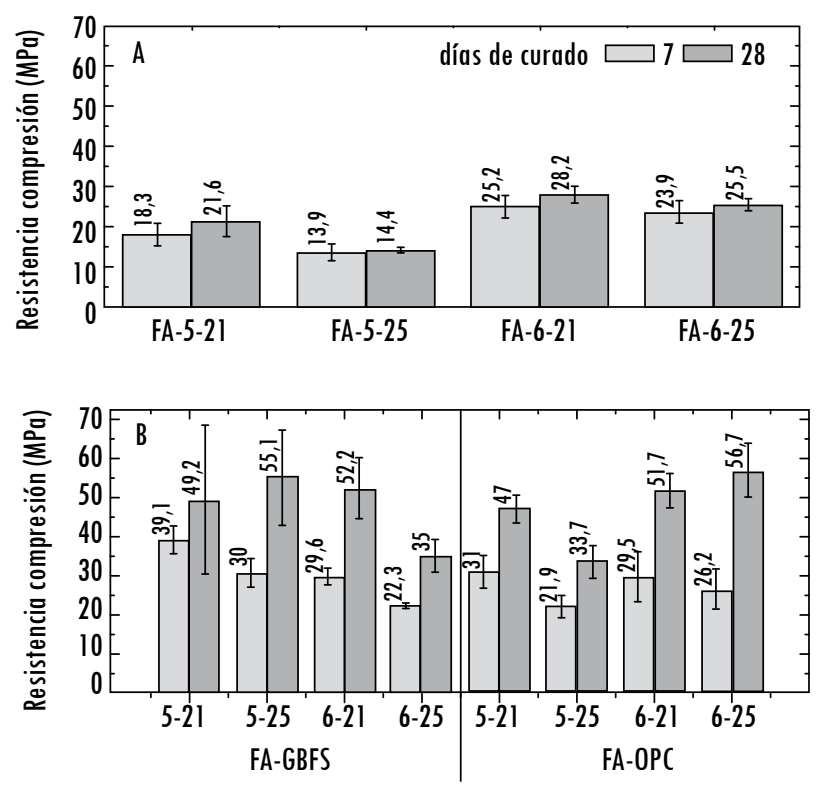

Fuente: presentación propia de los autores

Los resultados muestran un efecto más significativo de la relación $\mathrm{SiO}_{2} / \mathrm{Al}_{2} \mathrm{O}_{3}$ del sistema bajo las condiciones de activación del presente estudio para los geopolímeros FA, con incrementos de hasta un $37 \%$, en comparación con las relaciones $\mathrm{Na}_{2} \mathrm{O} / \mathrm{SiO}_{2}$, cuyo efecto sobre la resistencia a compresión puede ser considerado despreciable. Por otra parte, los valores de resistencia a 7 días de curado en los sistemas con una fuente de Ca (FA-OPC y FA-GBFS) exhibieron ligeros decrementos conforme se incrementan las relaciones molares $\mathrm{Na}_{2} \mathrm{O} / \mathrm{SiO}_{2}$ en el sistema (de 0,21 a 0,25). El incremento en la concentración de activación conduce a la presencia de una mayor cantidad de iones $\mathrm{OH}$ - que pueden atacar y 
romper los enlaces Si-O-Si, Si-O-Al y Al-O-Al de la fracción vítrea de la ceniza; sin embargo, el exceso de dichos iones puede contribuir a la disolución de los productos recientemente formados y tener un ligero efecto en el desempeño mecánico del material. No obstante, la presencia de especies activas de $\mathrm{SiO}_{2}$ mediante la incorporación de silicatos solubles reduce el tiempo necesario para la formación del gel a través de su interacción con las especies activas de Al liberadas durante la disolución y, por tanto, el aumento en el grado de polimerización del gel rico en $\mathrm{Si}$, que contribuye a una ganancia de resistencia mecánica después de 28 días de curado.

Al comparar los valores de resistencia a la compresión de los tres sistemas FA, FA/GBFS y FA/OPC a 28 días de curado con los de FA, es claro que tanto la GBFS como el OPC aportan al desarrollo mecánico y microestructural después de los 7 días de curado. El aumento de la resistencia mecánica en estos sistemas binarios puede atribuirse a la coexistencia del gel N-A-S-H obtenida tras la activación de la FA, junto con los geles C-S-H de la hidratación del OPC y el gel C-A-S-H formado tras la activación de la GBFS (García-Lodeiro, Fernández-Jiménez y Palomo, 2013; García-Lodeiro et al., 2010; Yip y Van Deventer, 2003; CabreraFuentes et al., 2011; Palomo et al., 2007).

El desempeño mecánico de los materiales evaluados en el presente estudio es coherente con lo reportado en la bibliografía para sistemas geopoliméricos basados en ceniza volante. No obstante, cabe resaltar que la gran variedad de resistencias mecánicas reportadas en la literatura es atribuida a la heterogeneidad del origen de las cenizas volantes, las cuales presentan diferencias en su composición química, mineralogía, contenido de fase vítrea y tamaño de partícula, así como en los diversos parámetros de activación y producción. Autores como Álvarez-Ayuso et al. (2009), Kovalchuk y Palomo (2008), entre otros, alcanzaron resistencias a compresión de hasta $60 \mathrm{MPa}$ con cenizas con pérdidas al fuego menores al $1 \%$. Por el contrario, Andini et al. [43], quienes activaron alcalinamente una FA con alto contenido de material orgánico $(\sim 11 \%)$ reportaron después de 5 días de curado una resistencia a compresión de 3,8 $\mathrm{MPa}$, valor notablemente inferior a la alcanzada en el presente estudio. Cabe anotar que este último es el único reporte donde se ha utilizado una ceniza con alto contenido de carbón no quemado en su composición.

\subsection{Microscopía electrónica de barrido}

La figura 4 muestra las micrografías de los sistemas FA-5-21, FA/OPC- 5-21 y FA/GBFS-5-21 después de 28 días de curado, en las cuales se puede identificar 
fácilmente la presencia de partículas de FA que no han reaccionado y que pueden encontrarse embebidas en el gel de los productos formados. Estas partículas de FA exhiben una forma esférica con el crecimiento de algunos productos de reacción en la superficie o la disolución parcial de la capa exterior, cuya microestructura interna revela la presencia de estructuras tubulares que pueden ser atribuidas a mullita (Lloyd, Provis y van Deventer, 2009). La obtención de una estructura porosa se atribuye a los espacios existentes entre el gel formado y las partículas de ceniza sin reaccionar. Así mismo, es posible observar otro tipo de partículas de diferente morfología, las cuales pueden atribuirse a las fuentes de Ca sin reaccionar. En términos generales, en las micrografías es posible observar la aparición de microgrietas generadas tanto por la retracción del material, lo cual es característico en sistemas geopoliméricos con relaciones $\mathrm{SiO}_{2} / \mathrm{Al}_{2} \mathrm{O}_{3}$ elevadas (Kamhangrittirong et al., 2013), como por el preacondicionamiento de las muestras antes de la realización del ensayo.

Figura 4. Análisis MEB de pastas geopoliméricas con 28 días de curado: a) FA, b) FA/OP( y c) FA/GBFS

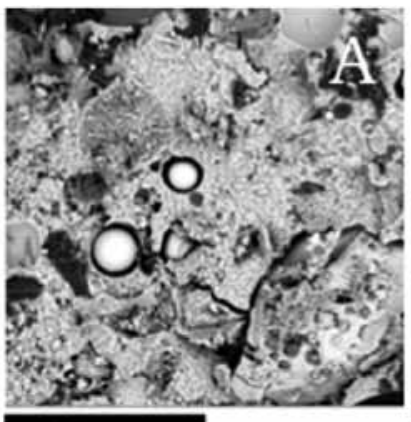

$30 \mu \mathrm{m}$

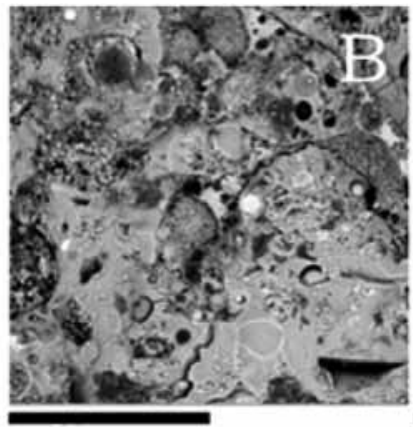

$30 \mu \mathrm{m}$

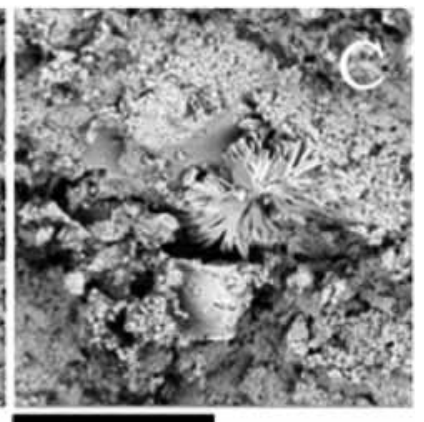

$30 \mu \mathrm{m}$

Fuente: presentación propia de los autores

\section{Conclusiones}

Los resultados obtenidos revelan la potencial utilización de una FA con alto contenido de pérdidas por ignición (14\%) como precursor en la fabricación de un material geopolimérico simple o binario. El preacondicionamiento de la ceniza mediante un tratamiento de molienda y su posterior activación alcalina con relaciones $\mathrm{SiO}_{2} / \mathrm{Al}_{2} \mathrm{O}_{3}$ y Na $2 \mathrm{O} / \mathrm{SiO}_{2}$ entre los rangos de 5-6 y 0,20-0,25, respectivamente, permiten la obtención de un material con características cementantes que puede adquirir resistencias de hasta $\sim 30 \mathrm{MPa}$ con 28 días de curado. La incorporación de una fuente de calcio (OPC o GBFS) al sistema de FA 
simple incrementó la resistencia a compresión a 28 días hasta tres veces alcanzando valores de hasta $\sim 56 \mathrm{MPa}$. La alta solubilidad del Ca en la escoria, bajo las condiciones en estudio condujo a una reducción de los valores de liberación de calor, la obtención de una estructura más densa y compacta que contribuye al incremento en el desempeño mecánico.

\section{Agradecimientos}

Los autores quieren dar su agradecimiento, por el apoyo en el desarrollo del presente estudio, a la Universidad del Valle (Cali, Colombia), al Centro de Excelencia en Nuevos Materiales (CENM), a la Termoeléctrica Colombiana GENSA y al Departamento Administrativo de Ciencia, Tecnología e Innovación (Colciencias).

\section{Referencias}

AHMARUZZAMAN, M. A review on the utilization of fly ash. Progress in Energy and Combustion Science. 2010, vol. 36, no. 6 pp. 327-363.

ALONSO, S. and PALOMO, A. Calorimetric study of alkaline activation of calcium hydroxidemetakaolin solid mixtures. Cement and Concrete Composites. 2001, vol. 31, no. 1, pp. 25-30.

ÁlVAREZ-AYUSO, E.; QUEROL, X.; PlANA, F.; ALASTUEY, A.; MORENO, N.; IZQUIERDO, M.; FONT, O.; MORENO, T.; DIEZ, S.; VÁZQUEZ, E. and BARRA, M. Environmental, physical and structural characterisation of geopolymer matrixes synthesised from coal (co-)combustion fly ashes. Journal of Hazardous Materials. 2009, vol. 154, no. 1-3, pp. 175-183.

ANDINI, S.; CIOFFI, R.; COLANGELO, F.; GRIECO, T.; MONTAGNARO, F. and SANTORO, L. Coal fly ash as raw material for the manufacture of geopolymer-based products. Waste Management. 2007, vol. 28, no. 2, pp. 416-423.

BERNAL, S. A.; PROVIS, J. L.; ROSE, V. and MEJÍA DE GUTIERREZ, R. Evolution of binder structure in sodium silicate-activated slag-metakaolin blends. Cement and Concrete Composites. 2011b, vol. 33, no. 1, pp. 46-54.

BERNAL, S. A.; RODRÍGUEZ, E. D.; MEJÍA DE GUTIÉRREZ, R.; PROVIS, J. L. and DELVASTO, S. Activation of metakaolin/slag blends using alkaline solutions based on chemically modified silica fume and rice husk ash. Waste and Biomass Valorization. 2011a, vol. 3, pp. 99-108.

CABRERA-FUENTES, B.; FERNÁNDEZ-JIMÉNEZ, A. A. and PALOMO, A. Alkaline activation of blended fly ash and cement kiln dust. Proceedings of 13 th International Congress on the Chemistry of Cement, Madrid, 2011, pp. 1-7.

CHITHIRAPUTHIRAN, S. and NEITHALATH, N. Isothermal reaction kinetics and temperature dependence of alkali activation of slag, fly ash and their blends. Construction and Building Materials. 2013, vol. 45, pp. 233-242. 
DUXSON, P.; FERNÁNDEZ-JIMÉNEZ, A.; PROVIS, J. L.; LUKEY, G. C.; PALOMO, A. and DEVENTER, J. S. J. Geopolymer technology: the current state of the art. Journal of Materials Science. 2007, vol. 42, no. 9, pp. 2917-2933.

DUXSON, P.; LUKEY, G. C.; SEPAROVIC, F. and VAN DEVENTER, J. S. J. Effect of alkali cations on aluminum incorporation in geopolymeric gels. Industrial E Engineering Chemistry Research. 2005, vol. 44, no. 4, pp. 832-839.

DUXSON, P.; PROVIS, J. L.; LUKEY, G. C.; SEPAROVIC, F. and VAN DEVENTER, J. S. J. 29Si NMR study of structural ordering in aluminosilicate geopolymer gels. Langmuir. 2005, vol. 21, no. 7, pp. 3028-3036.

FERNÁNDEZ-JIMÉNEZ, A.; PALOMO, A. and CRIADO, M. Microstructure development of alkali-activated fly ash cement: a descriptive model. Cement and Concrete Composites. 2005, vol. 35, no. 6, pp. 1204-1209.

FERNÁNDEZ-JIMÉNEZ, A. and PALOMO, A. Characterisation of fly ashes: Potential reactivity as alkaline cements. Fuel. 2003, vol. 82, no. 18, pp. 2259-2265.

FERNÁNDEZ-JIMÉNEZ, A.; SOBRADOS, I. and PALOMO, A. Hybrid cements with very low OPC content. Proceedings of 13th International Congress on the Chemistry of Cement, Madrid, July, 2011, pp. 1-7

GARCÍA-LODEIRO, I.; FERNÁNDEZ-JIMÉNEZ, A. and PALOMO, A. Hydration kinetics in hybrid binders: Early reaction stages. Cement and Concrete Composites. 2013, vol. 39, pp. 82-92.

GARCÍA-LODEIRO, I.; FERNÁNDEZ-JIMÉNEZ, A.; PALOMO, A. and MACPHEE, D. E. Effect of calcium additions on N-A-S-H cementitious gels. Journal of the American Ceramic Society. 2010, vol. 93, no. 7 pp. 1934-1940.

GIL, C. y PLAZAS, R. Propuesta para la utilización de cenizas volantes como adición en la fabricación de cemento tipo I en la planta cementera de Holcim Colombia S.A. Documento procedente de la Especialización en Gerencia de Producción y Operaciones, Universidad de La Sabana, Bogotá, 2007.

GRUYAERT, E.; ROBEYST, N. and DE BELIE, N. Study of the hydration of Portland cement blended with blast-furnace slag by calorimetry and thermogravimetry. Journal of Thermal Analysis E Calorimetry. 2010, vol. 102, no. 3, pp. 941-951.

IZQUIERDO, M.; QUEROL, X.; DAVIDOVITS, J.; ANTENUCCI, D.; NUGTEREN, H. and FERNÁNDEZ-PEREIRA, C. Coal fly ash-slag-based geopolymers: microstructure and metal leaching. Journal of Hazardous Materials. 2009, vol. 166, no. 1, pp. 561-566.

KAMHANGRITTIRONG, P.; SUWANVITAYA, P.; WITAYAKUL, W.; SUWANVITAYA, P. and CHINDAPRASIRT, P. Factors influence on shrinkage of high calcium fly ash geopolymer paste. Advanced Materials Research. 2013, vol. 610-613, pp. 2275-2281. 
KOVALCHUK, G.; FERNÁNDEZ-JIMÉNEZ, A. and PALOMO, A. Activación alcalina de cenizas volantes: relación entre el desarrollo mecánico resistente y la composición química de la ceniza. Materiales de Construcción. 2008, vol. 58, no. 291, pp. 35-52.

KUMAR, R.; KUMAR, S. and MEHROTRA, S. P. Towards sustainable solutions for fly ash through mechanical activation. Resources, Conservation and Recycling. 2007, vol. 52, no. 2, pp. 157-179.

KUMAR, S.; KUMAR, R. and MEHROTRA, S. P. Influence of granulated blast furnace slag on the reaction, structure and properties of fly ash based geopolymer. Journal of Materials Science. 2010, vol. 45, no. 3, pp. 607-615.

LEE, S.; SEO, M.-D.; KIM, Y.-J.; PARK, H.-H.; KIM, T.-N.; HWANG, Y. and CHO, S.-B. Unburned carbon removal effect on compressive strength development in a honeycomb briquette ash-based geopolymer. Int. International Journal of Mineral Processing. 2010, vol. 97 , no. $1-4$, pp. 20-25.

LLOYD, R.; PROVIS, J. and VAN DEVENTER, J. Microscopy and microanalysis of inorganic polymer cements. 2: the gel binder. Journal of Materials Science. 2009, vol. 44, no. 2, pp. 620-631.

NEMATOLLAHI, B. and SANJAYAN, J. Effect of different superplasticizers and activator combinations on workability and strength of fly ash based geopolymer. Materials $\mathcal{E}$ Design. 2014, vol. 57, pp. 667-672.

PALOMO, A.; FERNÁNDEZ-JIMÉNEZ, A.; KOVALCHUK, G.; ORDÓÑEZ, L. M. and NARANJO, M. C. OPC-fly ash cementitious systems: study of gel binders produced during alkaline hydration. Journal of Materials Science. 2007, vol. 42, no. 9, pp. 2958-2966.

PHOO-NGERNKHAM, T.; CHINDAPRASIRT, P.; SATA, V.; PANGDAENG, S. and SINSIRI, T. Properties of high calcium fly ash geopolymer pastes with Portland cement as an additive. Int. International Journal of Minerals, Metallurgy, and Materials. 2013, vol. 20, no. 2, pp. 214-220.

PROVIS, J. L. and VAN DEVENTER, J. S. J. Geopolymerisation kinetics: 2. Reaction kinetic modelling. Chemical Engineering Science. 2007, vol. 62, no. 9, pp. 2318-2329.

PROVIS, J. L. and VAN DEVENTER, J. S. J. Geopolymers: structures, processing, properties and industrial applications. Cambridge (UK): Woodhead Publishing Limited, 2009.

PUERTAS, F. and FERNÁNDEZ-JIMÉNEZ, A. Mineralogical and microstructural characterisation of alkali-activated fly ash / slag pastes. Cement and Concrete Composites. 2003, vol. 25, no. 3, pp. 287-292.

PUERTAS, F.; PALACIOS, M.; MANZANO, H.; DOLADO, J. S.; RICO, A. and RODRÍGUEZ, J. A model for the C-A-S-H gel formed in alkali-activated slag cements. Journal of the European Ceramic Society. 2011, vol. 31, no. 12, pp. 2043-2056. 
PUERTAS, F.; MARTÍNEZ-RAMÍREZ, S. and VÁZQUEZ, A. Alkali-activated fly ash / slag cement Strength behaviour and hydration products. Cement and Concrete Research. 2000, vol. 30, no. 10, pp. 1625-1632.

RAVIKUMAR, D. and NEITHALATH, N. Reaction kinetics in sodium silicate powder and liquid activated slag binders evaluated using isothermal calorimetry. Thermochimica Acta. 2012, vol. 546, pp. 32-43.

RYU, G. S.; LEE, Y. B.; KOH, K. T. and CHUNG, Y. S. The mechanical properties of fly ashbased geopolymer concrete with alkaline activators. Construction and Building Materials. 2013, vol. 47, pp. 409-418.

TEMUUJIN, J. and VAN RIESSEN, A. Effect of fly ash preliminary calcination on the properties of geopolymer. Construction and Building Materials. 2009, vol. 164, no. 2-3, pp. 634-639.

TEMUUJIN, J.; RICKARD, W. and VAN RIESSEN, A. Characterization of various fly ashes for preparation of geopolymers with advanced applications. Advanced Powder Technology. 2013, vol. 24, no. 2, pp. 495-498.

VAN DEVENTER, J. S.; PROVIS, J. J. L. and DUXSON, P. Technical and commercial progress in the adoption of geopolymer cement. Minerals Engineering. 2012, vol. 29, pp. 89-104.

VAN RIESSEN, A. and CHEN-TAN, N. Beneficiation of collie fly ash for synthesis of geopolymer Part 2 - Geopolymers. Fuel. 2013, vol. 111, pp. 829-835.

WINNEFELD, F.; LEEMANN, A.; LUCUK, M.; SVOBODA, P. and NEUROTH, M. Assessment of phase formation in alkali activated low and high calcium fly ashes in building materials. Construction and Building Materials. 2010, vol. 24, no. 6, pp. 1086-1093.

WORLD COAL ASSOCIATION. Coal statistics [document on line], 2014. < http://www. worldcoal.org/resources/coal-statistics/>

XU, G. Q.; HAN, D. L.; HAN, J. Q. and WANG, S. Research on the green concrete mixed the high-volume limestone powder and low-quality fly ash. Applied Mechanics and Materials. 2012, vol. 253, pp. 462-465.

XU, Q.; HU, J.; RUIZ, J. M.; WANG, K. and GE, Z. Isothermal calorimetry tests and modeling of cement hydration parameters. Thermochimica Acta. 2010, vol. 499, no. 1-2, pp. 91-99.

YIP, C. and VAN DEVENTER, J. Microanalysis of calcium silicate hydrate gel formed within a geopolymeric binder. Journal of Materials Science. 2003, vol. 38, no. 18, pp. 3851-3860.

YIP, C. K.; LUKEY, G. C.; PROVIS, J. L. and VAN DEVENTER, J. S. J. Effect of calcium silicate sources on geopolymerisation. Cement and Concrete Research. 2008, vol. 38, no. 4, pp. 554-564. 
